\title{
The importance of the intensity and frequency of perturbations on the germination delay
}

\author{
Alfonso Garmendia , Luis Garmendia , Adela Salvador \\ Department of Agro-forest Ecosystems, Mediterranean Agro-forest Institute, \\ Technical University of Valencia, E.T.S.M.R.E. (U.P.V.), Av. Blasco Ibánez 21, 46010 Valencia, Spain \\ Department of Programming and Computing Systems, Complutense University of Madrid, \\ Juan del Rosal 8, 28040 Madrid, Spain \\ Department of Applied Mathematics, Technical University of Madrid, E.T.S.I. Caminos, \\ Ciudad Universitaria sin. 28040 Madrid, Spain
}

\begin{abstract}
An iterative model is developed to evaluate the reproductive strategies of plants in environments with different frequencies and intensities of disturbance. Two extreme reproductive strategies are compared: the "homocarpic" strategy, in which all the seeds germinate the following spring, without dormancy, and the "heterocarpic" strategy, whereby, cach year, half of the existing seeds germinate. It is observed that this sort of heterocarpy is beneficial in environments with strong perturbations, in which a high pereentage of the population dies. However, the frequency of such perturbations does not cause changes in the advantage of one strategy over the other. The results suggest that the intensity of the disturbance is much more important than the frequency.
\end{abstract}

Keywords: Heterocarpy; Homocarpy; Germination strategy; Perturbation; Disturbance; Dormancy 


\section{Introduction}

This paper is the first part of a theoretical study about the evolution of heterocarpy in disturbed environments. The model tested the relative importance of intensity and frequency of disturbance to reproductive strategies of plants in variable environments. It has considered the transient dynamics and the patterns of two population co-existence, with two contrasting reproductive strategies. The population size is always an entire value, and therefore cannot be continuous, as was assumed in most previous works. Therefore, an iterative model would describe better the population dynamics that an analytic model based only on an equations system.

Heterocarpic species produce two or more types of seeds, combining in the seeds from one individual two or more divergent strategies. This strategy shows a tendency for seed functions to diverge, each type specializing in some aspect of environmental variation to which it is predisposed, while being buffered by the other seed types. Venable and Lawlor considered a species model with two kinds of seeds with different dispersability in space and time, and concluded that reproduction is maximized when one of them has high dispersability and a low depth of dormancy, and vice versa.

There are many heterocarpic or heteromorphic species and multiple germination strategies are very common. Many ecological studies had shown how they differed in within-year or betweenyear timing of germination and dispersal . Most of these studies have been done with the family Asteraceae, Chenopodiaceae or Brassicaceae . For instance, the betweenyear seed bank of Atriplex triangularis (Chenopodiaceae) differed in the large, brown, easily germinable, and the small, black, less germinable forms of its seeds . Seeds of other species, e.g. Heterosperma pinnatum (Asteraceae), did not over-winter and the ability to germinate did not differ between peripheral and central achenes. In these cases, within-year timing of germination may be very important

Under stressful conditions, the varying dispersal distances and levels of dormancy associated with heterocarpy may enhance the probability of germination and/or seedling survival , but how stressful does the environment have to be for heterocarpy to be worthwhile?

Theoretical studies dealing with delayed germination beyond one year, referred to this as a "bet-hedging" adaptation to the unpredictability of desert environments or explained it as an escape from the negative effect of density or sibling competition, prevent mortality under high densities by delayed germination . Most of these theoretical models were constructed by equations that predicted the long-term expected values for population size, but did not deal with the variability or the fluctuations, although it is an important aspect of empirical studies. Cohen used $Y$ as the average number of seeds per germinating seedling. $Y$ then was a random variable depending on environmental conditions, and was assumed to be independent of the population density. Also used $P_{y^{\prime}}$ as the probability of having a yield $Y$. He expected the fraction that germinates every year to be approximately equal to the probability of producing a high yield, and the fraction that does not germinate to be also approximately equal to the probability of total or near total failure to produce seeds.

There are many studies that use the "level of disturbance" as a parameter to predict diverse population patterns. In several cases this level of disturbance was only defined as a unique value, representing its frequency, its intensity or a combination of both. In this work, disturbance 
is defined by two variables: the probability of occurrence $(O P)$ and the perturbation intensity $(P I)$. These two variables were random and independent in our model, and were fixed the beginning of each model experiment. The relative importance of these two variables was determined for two ideal plant populations with different strategies for survival: The homocarpic strategy population had all seeds germinating in the first year, and therefore, did not form a seeds bank. The heterocarpic strategy population formed a seed bank from which only half of the seeds germinated each year. The model tested the conditions that favour these specific strategies.

The number of adult plants that fit within a given territory was limited and, therefore, plants competed for suitable sites. Consequently, the maximum number of plants corresponded to the carrying capacity of the environment. In order to isolate the variables studied, the number of seeds produced by each plant, and other population variables (independent variables) were assumed the same for both populations.

For some types of perturbation, it may be necessary to calculate the possibility of more than one perturbation within a year, and $O P$ should be used as the parameter for 'annual rate of perturbations' of a Poisson distribution. In this case this has not been taken into account and only can be one disturbance by year. This can be useful, for example, to model drought perturbations.

Perturbations were the only causes of death considered for adult plants. Other possible causes of death, such as natural death (longevity), were not considered in order to eliminate the possible noise of distinct death types, therefore, the model fits for perennial aged plants. In addition, the effect of disturbances on the seeds or seedlings had not been taken into account, because it was supposed that it affected equally to both species and did not change seeds or seedlings proportion. It is proved that heterocarpy is a competitive strategy for perturbed environments, because the heterocarpic population dominated in those environments with high values of $P I$ and $O P$, whereas the homocarpic population dominated for the lowest values for these variables. For some $P I$ and $O P$ values, an equilibrium point was identified between homocarpy and heterocarpy.

\section{Materials and methods: The model}

The model has been implemented using a $\mathrm{C}++$ program in which the territory was a toroidal of $n \times n$ cells to avoid the presence of borders.

Six independent and seven dependent variables have been taken into account. The independent variables were: (1) the time in years, $t$; (2) the number of seeds spread per plant, $s p$; (3) the maximum number of plants per cell, $m p$; (4) the number of initial seeds per cell, $s_{0} ;(5)$ the probability of a perturbation occurring, $O P$; and (6) the perturbation intensity, $P I$. The dependent variables were: (1) $s(i, j, t)$, the number of seeds that fell during the year $t$ in each $(i, j)$ cell; $(2) S_{H o}(i, j, t)$, the number of seeds belonging to the homocarpic population, in each $(i, j)$ cell, during the year $t ;(3)$ $S_{H E}(i, j, t)$, the number of seeds belonging to the heterocarpic population, in each $(i, j)$ cell, during the year $t$; (4) $P A_{H O}(i, j, t)$, the number of adult homocarpic plants, in each $(i, j)$ cell, during the year $t ;(5) P A_{H E}(i, j, t)$, the number of adult heterocarpic plants, in each $(i, j)$ cell, during the year $t ;(6) H(i, j, t)$, the number of free suitable sites for adult plants, in each $(i, j)$ cell, during the year $t$; and (7) $\alpha(i, j, t)$, the probability of a homocarpic seed becoming an adult plant, in each $(i, j)$ cell, during the year $t$. 


$$
\begin{aligned}
& S_{H O}(i, j, t)=f\left(s p, P A_{H O}(i, j, t), P A_{H O}(i-1, j-1, t), P A_{H O}(i-1, j, t), P A_{H O}(i-1, j+1, t),\right. \\
& P A_{H O}(i, j-1, t), P A_{H O}(i, j+1, t), P A_{H O}(i+1, j-1, t), P A_{H O}(i+1, j, t), \\
&\left.P A_{H O}(i+1, j+1, t)\right),
\end{aligned}
$$

and

$$
\begin{aligned}
S_{H E}(i, j, t)=f\left(s p, P A_{H E}(i, j, t), P A_{H E}(i-1, j-1, t), P A_{H E}(i-1, j, t), P A_{H E}(i-1, j+1, t),\right. & \\
& P A_{H E}(i, j-1, t), P A_{H E}(i, j+1, t), P A_{H E}(i+1, j-1, t), P A_{H E}(i+1, j, t), \\
& \left.P A_{H E}(i+1, j+1, t)\right) .
\end{aligned}
$$

Each year, all the seeds from the homocarpic population germinate, but only half of the heterocarpic population seeds germinate in the first year. The remaining seeds do not germinate and are accumulated in a seed bank. In the following years, half of the heterocarpic seeds in the bank germinate. A germination factor $G$ could have been considered, but was omitted in order to avoid an increase in the number of variables, which could have produced unclear results. Also, it should be considered that a certain number of seeds die each year. However, this factor is accounted for by using a smaller number of seeds produced per plant.

Two random and independent variables were considered to define a perturbation: the occurrence probability of a perturbation $O P$, and the intensity of this perturbation $P I$. Disturbances may occur every year (high $O P$ ), or may occur only once over several years (low $O P$ ). However, these can be of a high or low intensity. With low-intensity perturbations, only a few individuals die, whereas with high intensities, a great number die. $P I$ represented the probability of dying for each individual plant during a perturbation episode. These two probabilities were considered according to the methods used to evaluate the risk of accidents: in the evaluation of the accident risk of a road are considered both, the probability of an accident occurring, and the probability of that accident being severe. They are also very similar to the yield $Y$ and $P_{y}$ used by Cohen [21].

In the model, the perturbations affected only adult plants, and it was the only cause of death considered. It seems obvious that the results can vary greatly whether annual plants or plants with great longevity are considered, although, perhaps this only means that the same dynamics are simply slowed. Subsequent studies may include new independent variables: the maximum number of years that each plant, seedling and seed can live. In this study, those variables were eliminated to concentrate on those under consideration. Any causes of death for adult plants other than the perturbations were not included in this first algorithm. Concerning the seedlings, once a seed germinates, if there was not a free site in its cell, it died.

In other words, for an adult plant to die, a perturbation had to occur $(p=O P)$, and the plant has to be affected $(p=P I)$. This means that the probability of dying for an adult plant was $O P * P I$ and, therefore, the probability of an adult plant surviving in a given year was: 1 $O P * P I$, and this probability has been assumed to be the same for both populations. It is important not to confuse this probability with the proportion of plants dying each year. If the perturbation did not occur, any adult plant died. These probabilities were implemented with the function random of $\mathrm{C}++$.

The maximum number of plants per cell was fixed as $m p$. A proportional competition algorithm is proposed for calculating, for each cell and each year, the probability $\alpha(i, j, t)$ as the proportion of homocarpic seeds from the total number of seeds to germinate. Since the total number of seeds 
that will germinate in one $(i, j)$ cell was the total number of homocarpic seeds and half of the heterocarpic seeds placed in that cell, then $\alpha(t)$ equals, for the year $t$ and in the $(i, j)$ cell, the total number of homocarpic seeds of that cell divided by the total number of seeds that germinate from both populations: $\alpha(i, j, t)=S_{H O}(i, j, t) /\left(S_{H O}(i, j, t)+S_{H E}(i, j, t) / 2\right)$. This probability was applied to each homocarpic seed for each existing free space in the cell. Similarly, the probability of a heterocarpic plant occupying this place was $1-\alpha(i, j, t)$. Using this proportional algorithm, the probability of extinction of a population was lower than when using the algorithm of probability 0.5 .

The number of free sites was calculated for each cell and each year as the maximum number of plants per cell, $m p$, minus the number of plants of both populations in each cell during year $t$. Therefore:

$$
H(i, j, t)=m p-P A_{H O}(i, j, t)-P A_{H E}(i, j, t) .
$$

Taking this into account, the model used for each $(i, j)$ cell was:

\subsection{Init}

$$
\begin{aligned}
& S_{H O}(i, j, 0)+S_{H E}(i, j, 0)=s(i, j, 0)=s_{0}, \\
& P A_{H O}(i, j, 0)=0, \\
& P A_{H E}(i, j, 0)=0,
\end{aligned}
$$

\subsection{Algorithm}

$$
\begin{aligned}
& S_{H O}(i, j, t+1)=s_{H O}(i, j, t), \\
& S_{H E}(i, j, t+1)=s_{H E}(i, j, t)+\frac{1}{2} * S_{H E}(i, j, t), \\
& s(i, j, t+1)=S_{H O}(i, j, t+1)+S_{H E}(i, j, t+1) \\
& P A_{H O}(i, j, t+1)=(1-O P * P I) *\left(P A_{H O}(i, j, t)+\alpha(i, j, t) * H(i, j, t)\right) \\
& P A_{H E}(i, j, t+1)=(1-O P * P I) *\left(P A_{H E}(i, j, t)+(1-\alpha(i, j, t)) * H(i, j, t)\right),
\end{aligned}
$$

being

$$
\alpha(i, j, t)=S_{H O}(i, j, t) /\left(S_{H O}(i, j, t)+S_{H E}(i, j, t) / 2\right),
$$

and

$$
H(i, j, t)=m p-P A_{H O}(i, j, t)-P A_{H E}(i, j, t) .
$$

The program was run with distinct values for all the independent variables. It was observed that the main final results and conclusions were not changed. For the results exposition, the following values were taken for the independent variables:

Number of cells: $10 \times 10$

Maximum number of plants per cell: 5

Initial maximum number of seeds per cell: 8

Number of seeds per plant: 3 
Also, different values for $P I$ and $O P$ were used in the program during long time series $(1000$ years), and the mean values, medians, sums, root-mean-square deviations, ranks and quartiles for 100 years, 200 years and 1000 years are noted for the seeds and for the plants of both populations.

The total number of seeds and plants of each population after 1000 years, ignoring the first 100 years, for a fixed occurrence probability of perturbation $O P=0.5$ and $P I$ from 0 to 1 in 0.1 intervals, was analyzed 10 times for each $P I$, and the mean value of the 10 sequences obtained for each $O P$ and $P I$ was calculated.

The maximum number of plants permitted in each cell was five. Therefore, the maximum number of plants per year was limited to 500 . Also, eight was the initial maximum number of seeds spread per cell; therefore, the maximum number of initial seeds was 800. Each year, each adult plant produced three seeds and, therefore, the maximum number of seeds was:

$$
1500+800=2300 .
$$

\section{Results}

When the program was run with different initial values for the independent variables, distinct relations were found between these and the dependent variables: perturbation intensity $(P I)$ and probability of a perturbation ( $O P$ ) with the total number of seeds and plants, the relative importance of $P I$ and $O P$ and the two strategies response to $P I$. Also the relation between resilience and the seed bank is explained.

\subsection{Total number of seeds and plants}

It was observed that the perturbation intensity $(P I)$ affected the total number of seeds and plants more than the probability of a perturbation $(O P)$ occurring. For a fixed $P I$ there were not significant differences for distinct $O P$ values.

It can be observed that there was a strong linear fit between the sum of the seeds of both populations and the perturbation intensity (see Fig. 1 and Table 1). The mean value of the sum of the

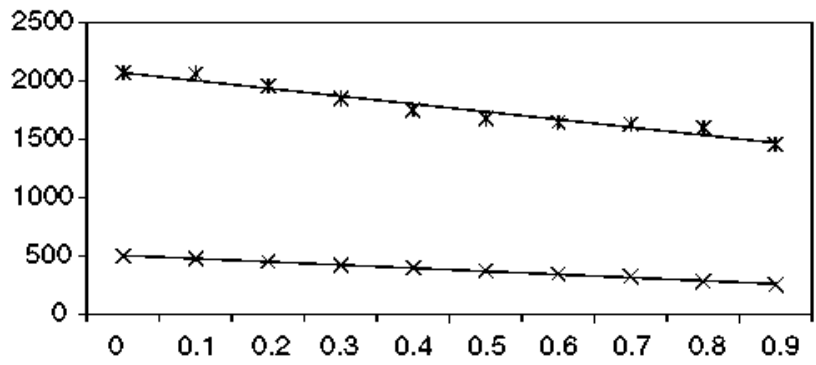

Fig. 1. Effect of perturbation intensity $(P I)$ on the average value of 10 sequences during 1000 years, on the number $(n)$ of plants (grey) and seeds (black) of both populations. $O P=0.5$ in all cases. 
Table 1

Statistics for different values of perturbation intensity ( $P I)$ with a 0.5 probability of perturbation occurrence $(O P)$

\begin{tabular}{llllllllllll}
\hline$P I$ & 0 & 0.1 & 0.2 & 0.3 & 0.4 & 0.5 & 0.6 & 0.7 & 0.8 & 0.9 & 1 \\
\hline HE & 207.2 & 228.8 & 218.9 & 210.4 & 202.0 & 204.2 & 218.1 & 237.6 & 269.8 & 250.1 & 32.7 \\
HO & 292.1 & 245.8 & 231.8 & 212.4 & 196.6 & 167.5 & 130.2 & 85.0 & 11.3 & 3.2 & 0.8 \\
Total plants & 499.3 & 474.6 & 450.7 & 422.8 & 398.6 & 371.7 & 348.3 & 322.7 & 281.1 & 253.3 & 33.4 \\
SD plants & $(0.06)$ & $(0.62)$ & $(1.06)$ & $(1.40)$ & $(2.48)$ & $(3.65)$ & $(5.23)$ & $(5.36)$ & $(47.45)$ & $(7.48)$ & $(32.69)$ \\
$100 \mathrm{HE}$ & 207.5 & 231.5 & 217.2 & 201.4 & 188.5 & 223.1 & 207.5 & 230.1 & 274.4 & 258.4 & 0 \\
$100 \mathrm{HO}$ & 292.5 & 249.9 & 232 & 206.9 & 189.7 & 176.9 & 111 & 91.1 & 23.5 & 3.6 & 0 \\
$200 \mathrm{HE}$ & 207.5 & 220.5 & 207.2 & 225.1 & 189 & 208.3 & 221.7 & 301.1 & 354.4 & 147.4 & 0 \\
$200 \mathrm{HO}$ & 292.5 & 250.5 & 210 & 217.3 & 189 & 167.9 & 132.4 & 127 & 16.3 & 0 & 0 \\
$1000 \mathrm{HE}$ & 207.5 & 228.8 & 218.6 & 239.4 & 171.9 & 185 & 212.9 & 246.8 & 379.8 & 275.4 & 0 \\
$1000 \mathrm{HO}$ & 292.5 & 250.8 & 229.6 & 234 & 166.6 & 186.3 & 108.7 & 68.6 & 0 & 0 & 0 \\
Seeds HO & 877.5 & 725.4 & 737.4 & 698.7 & 580.2 & 590.4 & 389.1 & 186.3 & 0 & 0 & 0 \\
Seeds HE & 1188.9 & 1331.8 & 1218.7 & 1147.7 & 1169.2 & 1084.8 & 1257.4 & 1442.3 & 1598.4 & 1457.6 & 190.8 \\
Total seeds & 2066.4 & 2057.2 & 1956.1 & 1846.4 & 1749.4 & 1675.2 & 1646.5 & 1628.6 & 1598.4 & 1457.6 & 190.8 \\
SD seeds & $(45.6)$ & $(2.0)$ & $(7.1)$ & $(8.4)$ & $(13.1)$ & $(15.2)$ & $(24.3)$ & $(36.9)$ & $(43.0)$ & $(47.6)$ & $(190.2)$ \\
\hline
\end{tabular}

$H E$ and $H O$ are the adult plants average population size of the heterocarpic and homocarpic populations, respectively, of 10 sequences during 1000 years, excluding the first 100 years. Total plants is the average sum of both populations' sizes. $100 \mathrm{HE}, 100 \mathrm{HO}, 200 \mathrm{HE}, 200 \mathrm{HO}$ and $1000 \mathrm{HE}, 1000 \mathrm{HO}$ values represent the average value of the 10 sequences for the population sizes in that year. Seeds HO and seeds HE are the average ( 10 sequences) number of homocarpic and heterocarpic seeds of the year 1000 , respectively. Total seeds is the average sum of the number of seeds. SD represents the standard deviations in both cases.

seeds for the 10 sequences, of both populations, per year, produced over 1000 years, except for the first 100 years, adjusted to:

Sum of seeds $=-66.7 * P I+2134.9$.

The mean value of the total sum of the plants from both populations produced over 900 years (1000 years, minus the first 100 years), and for the 10 sequence studies, adjusted to:

Sum of the plants $=-27.0 * P I+530.9$.

The typical deviation of these mean values was very small.

The correlation coefficients $(\rho)$ were very high in terms of their absolute value: $\rho=-0.997$ for $P I$ and the sum of plants, and $\rho=-0.979$ for $P I$ and the sum of seeds. Therefore, the goodness of fit of the linear regression was high.

\subsection{Relative importance of $P I$ and $O P$}

The behaviour of homocarpic and heterocarpic populations in a time series of 1000 years, with competition for the territory, with different $O P$ and $P I$ values, was analysed as (Fig. 2): for a $P I=0.2$ and any value of $O P$, the amplitude of the oscillations was similar, (approximately 100 plants), with a very similar time series. It was observed also that, in all cases, the number of homocarpic plants oscillated between 200 and 300 , whereas the number of heterocarpic plants 


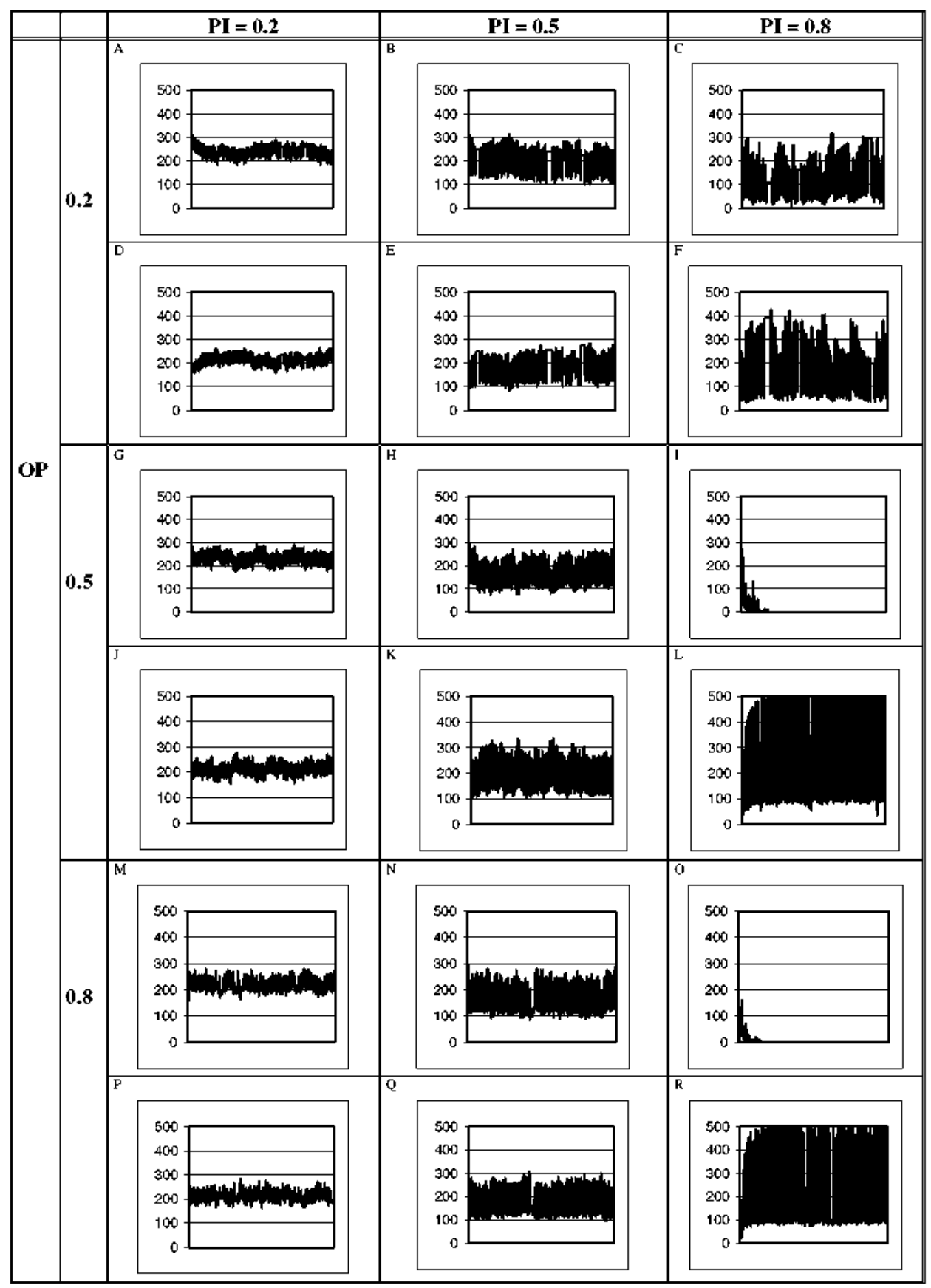

Fig. 2. A 1000 year temporal series for different perturbation occurrence probability $(O P)$ and perturbation intensities $(P I)$. The upper graph corresponds to the homocarpic population (A-C, G-I, M-O) and the lower to the heterocarpic population (D-F, J-L, P-R). Each pair of graphs represents only one particular case, but it is easy to see the growing fluctuations in amplitude with higher perturbation intensities. 


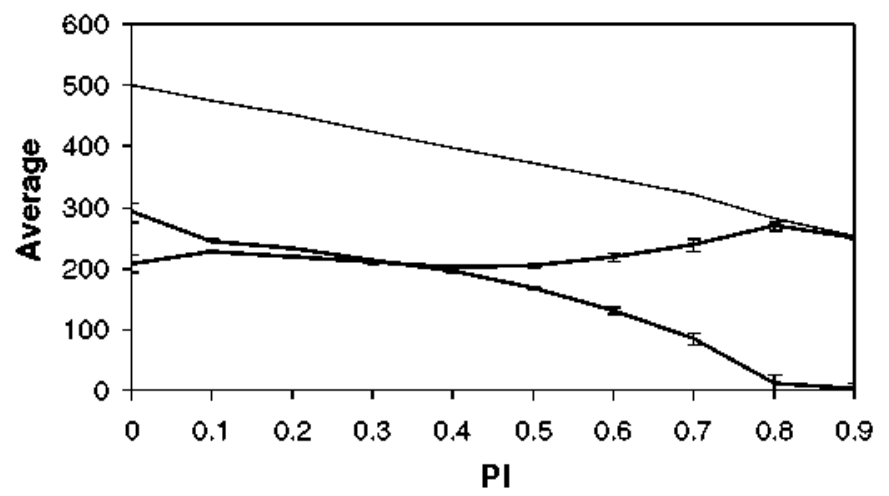

Fig. 3. Effect of the perturbation intensity $(P I)$ on the mean population sizes $n$ of the total plant number (grey area), heterocarpic ( $H E$, grey line) and homocarpic ( $H O$, black line) plants for 10 temporal series during 1000 years. The probability of perturbation occurrence $(P O)$ is 0.5 in all cases. The standard deviations are given.

oscillated between approximately 150 and 250 . For $P I=0.5$ and any given value of $O P$, the amplitude of the oscillations was approximately 150 plants, and the number of homocarpic and heterocarpic plants oscillated between 100 and 250 . When $P I=0.8$ and $O P=0.5$ or $O P=0.8$, homocarpic plants became extinct and, therefore the number of heterocarpic plants increased, with much greater oscillation amplitudes, from values of around 100 plants to 500 plants (maximum). The seed bank maintained by the heterocarpic population favoured this strategy for high $P I$ values. For $P I=0.8$ and $O P=0.2$, it was observed that the amplitude of the oscillations increased, and the mean value of heterocarpic plants was higher than that of the homocarpic plants. The resilience of heterocarpic plants was high due to the high number of seeds maintained in the seed bank.

From these results, it was concluded that, although the probability of a perturbation occurring is important, the intensity is the determining factor for the behaviour of species with contrasting strategies for survival: i.e. heterocarpic versus homocarpic.

\subsection{Homocarpic and heterocarpic response to the intensity of a perturbation}

The correlation coefficient between the perturbation intensity, $P I$, and the number of seeds and plants belonging to the homocarpic population was very high, but negative $(-0.98$ both). The correlation coefficient between the mean values of the number of seeds and plants of the heterocarpic population was also very high and negative; -0.91 for the seeds and -0.89 for the plants. When $P I$ increased, the mean values for seeds and adult plants diminished, but for low perturbation intensities, the homocarpic strategy presented better results than the heterocarpic strategy and for high intensities, the heterocarpic strategy population dominated. It was possible to find an equilibrium point between these strategies. In the particular case of Fig. 3, for $O P=0.5$, the equilibrium point was at $P I=0.3$. For these values, both strategies were balanced, and the homocarpic population presented better results for a lower $P I$ and the heterocarpic population presented better results for a higher $P I$. When different population values are used, the point of equilibrium 
between both strategies can change, but there is always a $P I$ value after which the heterocarpic strategy proves superior to the homocarpic strategy.

The mean population size values of the homocarpic plants adjusted to:

Homocarpic plants $=-31.8 * P I+332.6$.

Heterocarpic plants were more resilient and their number depended on $P I$, and on the number of homocarpic plants (competition). PI had a double effect; a negative effect on adult survival, and a positive effect, based on competition, due to the lower number of homocarpic plants. When the homocarpic plants diminished due to $P I$, the number of heterocarpic plants increased (see Fig. 3 ). Therefore, the adjustment of the heterocarpic population was poorer:

Heterocarpic plants $=4.8 * P I+198.3$.

\subsection{Resilience and seed bank}

The higher resilience observed for heterocarpic strategies can be explained by the seed permanence in the soil seed bank. As $P I$ increased, total plant and seed number decreased but, although perturbation affected adult plants, the re-colonization (competition) depended on the soil seed bank. In a low $P I$ scenario, the number of germinating seeds was higher for homocarpic plants than for heterocarpic plants, although the total seed number was similar (only half of heterocarpic seeds germinate). But for a high $P I$ scenario, the death of a high percentage of adult plants produced a decrease of the number of homocarpic plant seeds and, therefore, a lowering of competition ability at the next time-step. This favoured the heterocarpic strategy, which increased the number of adult plants and therefore its seed production. The differing slopes of the trend lines in Fig. 4 reflect that when perturbation intensity increased, besides total number of seeds decreased linearly, it was caused by the linear decreasing number of homocarpic seeds. At the same time, the amount of heterocarpic seeds had a soft increase as a result of the competition reduction.

In other words, the competition capability of these strategies after a perturbation is what was conditioning the total competition process. It was after a perturbation when gaps were open and, therefore, the number of seeds was important for competition. If there was no perturbation or it

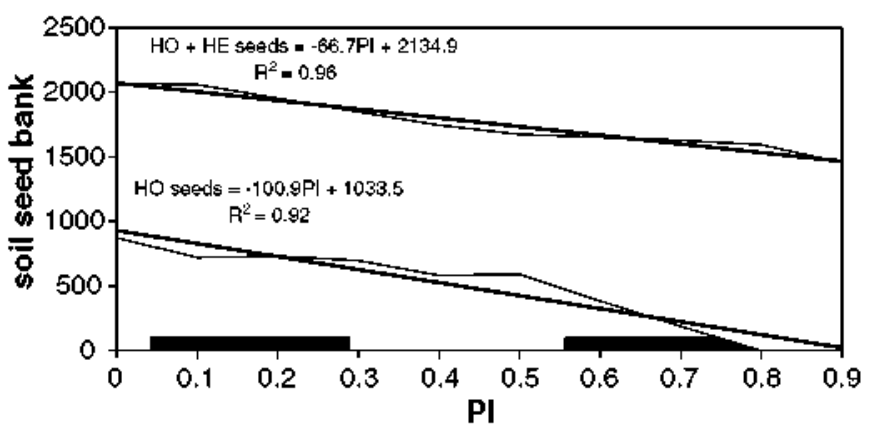

Fig. 4. The effect of the perturbation intensity $(P I)$ on the mean seed number in the soil seed bank: HO seeds number, dark grey; total seed number, light grey. The data are for 10 temporal series during 1000 years. The probability of perturbation occurrence $(P O)$ is 0.5 in all cases. Trend lines are shown. 
was of low intensity, there was none or very few gaps to colonize and most germinated seeds die. The heterocarpic strategy maximized the number of seeds germinating after a perturbation, while the homocarpic strategy produced more germinating seeds in time-steps without perturbation and fewer germinating seeds in the after-perturbation time-steps, when gaps were open.

\section{Conclusions}

A heterocarpic strategy has more resilience and, therefore, shows better results than the homocarpic strategy in disturbed environments. The opposite is true in virtually stable or more predictable environments, in which the heterocarpic strategy does not present any advantage. Indeed, the delay in the germination process can be a disadvantage in such environments. This observation coincides with the results obtained by other theoretical models and empirical observations of several species or different taxonomic groups . Nevertheless, the equilibrium between these two strategies depends on the perturbation intensity, regardless of its frequency.

The results suggest that the disturbance intensity is much more important than its frequency. Therefore, the heterocarpic strategy is an adaptation that can be very important for survival in places where disturbances are capable of killing a high percentage of adult plants, almost regardless of whether these perturbations are very frequent or infrequent. In fact, only in cases where the perturbations are extremely severe (with mortalities of around $80 \%$ each time they are produced), is the frequency with which these disturbances occur of some significance. When the perturbations are less intense, the equilibrium point between the homocarpic and heterocarpic strategies depends only on the perturbation intensity and does not change (or changes only slightly) according to their frequency.

Regarding the above, a prediction of this model is that disregarding the frequency with which disturbances occur, for an environmental gradient of a lower to greater intensity of these perturbations, a low PI habitat will support few heterocarpic strategy species, and the favourable strategy will be homocarpy, whereas a site of intense perturbations will be dominated by heterocarpic strategy species. There will always be an intermediate equilibrium point for the perturbation intensity, at which neither of these strategies will dominate over the other, but they will co-exist in a dynamic equilibrium. This is consistent with the results obtained by Cohen with differential equations.

In nature, unlike in the theoretical model, the intensity of the disturbances is not always the same. For example, droughts of greater or lower severity can occur. In these cases only the maximum disturbance intensity produced in each place needs to be considered. Even if the frequency of these maximum intensity perturbations is very low, the intensity is the variable capable of producing an effect. Therefore, this maximum disturbance intensity, and the re-colonization capability after a strong disturbance, determines the equilibrium between homocarpy and heterocarpy in natural populations.

Obviously, there are other environmental factors that will have an effect on both strategies: the genetic characteristics of the species or the historical characteristics of the place in which they live. Subsequent studies may analyse the effect of other factors, such as the seed and/or seedling mortality, which have not been taken into account in this first model. Nevertheless, it will be interesting to distinguish between distinct types of perturbation. Some perturbations, such as heavy frosts 
or floods, will have an $O P$ calculated as the parameter for annual rate of perturbations of a Poisson distribution. For fire events, $O P$ should decrease to a low value after the perturbation (low combustible) and increase with time from the last fire. The combined effects of different perturbations could also be calculated.

\section{References}

D.L. Venable, The evolutionary ecology of seed heteromorphism, American Naturalist 126 (1985) 577.

B. Mandak, Germination requirements of invasive and non-invasive Atriplex species: a comparative study, Flora 198 (2003) 45.

D.L. Venable, L. Lawlor, Delayed germination and dispersal in desert annuals: escape in space and time, Oecologia $46(1980) 272$.

G.A. Baker, D.J. O'Dowd, Effect of parent plant density on the production of achene types in the annual Hypochoeris glabra, Journal of Ecology 70 (1982) 201.

B.D. Tanowitz, P.F. Salopek, B.E. Mahall, Differential germination of ray and disc achenes in Hemizonia increscens (Asteraceae), American Journal of Botany 74 (1987) 303.

H. Yamaguchi, K. Ichilhara, K. Takeno, Y. Hori, T. Saito, Diversities in morphological characteristics and seed germination behaviour in fruits of Salsola korovii Iljin, Botanical Magazine 103 (1990) 177.

J. Kigel, Diaspore heteromorphism and germination in populations of the ephemeral Hedypnois rhagadioloides, (L.) F.W. Schmidt (Asteraceae) inhabiting a geographic range of increasing aridity, Acta Oecologica 13 (1992) 45.

E.R. De Clavijo, Heterocarpy and seed polymorphism in Ceratocapnos heterocarpa (Fumariaceae), International Journal of Plant Science 155 (1994) 196.

E.R. De Clavijo, The ecological significance of fruit heteromorphism in the amphicarpic species Catananche lutea (Asteraceae), International Journal of Plant Science 156 (1995) 824.

B. Mandak, P. Pysek, Fruit dispersal and seed banks in Atriplex sagittata: the role of heterocarpy, Journal of Ecology 89 (2001) 59.

D. Koller, N. Roth, Studies on the ecological significance of amphicarpy in Gymnarthena micrantha (Compositae), American Journal of Botany 5 (1964) 26.

A.E. Sorensen, Somatic polymorphism and seed dispersal, Nature 276 (1978) 174.

P.B. McEvoy, C.S. Cox, Wind dispersal distance in dimorphic achenes of ragwort, Senecio jacobea, Ecology 68 (1987) 2006.

O.J. Rocha, The effects of achene heteromorphism on the dispersal capacity of Bidens pilosa L, International Journal of Plant Science 157 (1996) 316.

B. Mandak, P. Pysek, The effects of light quality, nitrate concentration, and presence of bracteoles on germination of different fruit types in the heterocarpous Atriplex sagittata, Journal of Ecology 89 (2001) 149.

B. Mandak, Seed heteromorphism and the life cycle of plants: a literature review, Preslia 69 (1997) 129.

M.L. Stanton, Development and genetic sources of seed weight variation in Raphanus raphanistrum L. (Brassicaceae), American Journal of Botany 71 (1984) 1090.

B.A. Wertis, I.A. Ungar, Seed demography and seedling survival in a population of Ariplex triangularis Willd, American Midland Naturalist 116 (1986) 152.

D.L. Venable, E. Dyreson, E. Morales, Population dynamics consequences and evolution of seed traits of Heterosperma pimatum (Asteraceae), American Journal of Botany 82 (1995) 410.

I.A. Ungar, Population ecology of halophyte seeds, Botanical Review 53 (1987) 301.

D. Cohen, Optimizing reproduction in a randomly varying environment, Journal of Theoretical Biology 12 (1966) 119.

D. Cohen, Optimizing reproduction in a randomly varying environment when a correlation may exist between the conditions at the time a choice has to made and the subsequent outcome, Journal of Theoretical Biology 16 (1967) 1.

D.L. Venable, Modelling the evolutionary ecology of seed banks, in: M.A. Leck, V.T. Parker, R.L. Simpson (Eds.), Ecology of Soil Seed Banks, Academic Press Inc., San Diego, 1989, pp. 67-87. 
T. Philippi, Bet-hedging germination of desert annuals: beyond the first year, American Naturalist 142 (1993) 474. T. Philippi, Bet-hedging germination of desert annuals: variation among population and maternal effects in Lepidium lasiocarpum, American Naturalist 142 (1993) 488.

D.L. Venable, J.S. Brown, The population-dynamic functions of seed dispersal, Vegetation 107/108 (1993) 31.

S.P. Ellner, ESS germination strategies in randomly varying environments I. Logistic type models, Theoretical Population Biology 28 (1985) 50 .

S.P. Ellner, Germination dimorphism and parent-offspring conflict in seed germination, Journal of Theoretical Biology 123 (1986) 173.

S.P. Ellner, Competition and dormancy: a reanalysis and review, American Naturalist 130 (1987) 798.

S.D. Levin, D. Cohen, A. Hastings, Dispersal strategies in patchy environment, Theoretical Population Biology 26 (1984) 165.

D.J. Schoen, D.G. Lloyd, The selection of cleistogamy and heteromorphic diasporas, Biological Journal of Linnean Society 23 (1984) 303.

Andrew R. Dyer, Maternal and sibling factors induce dormancy in dimorphic seed pairs of Aegilops trituncialis, Plant Ecology 172 (2) (2004) 211. 